\title{
Bilinear System Control with Exponential Stability
}

\author{
Min-Shin Chin \\ Department of Mechanical Engineering \\ National Taiwan University \\ Taipei, Taiwan, Republic of China \\ TEL:02-23630231 ext. 2414 \\ FAX:02-23631755 \\ EMAIL:mschen@ccms.ntu.edu.tw
}

\begin{abstract}
For a bilinear system that is open-loop neutrally stable, a quadratic state feedback control has been proposed to ensure global asymptotical stability of the closed-loop system. In this paper, a new nonlinear control is proposed so that the closed-loop system is not only globally stable but also exponentially stable. The new control results in a much faster state convergence rate; furthermore, it can be applied to a constrained bilinear system which is subject to whatever tight saturation limits on the control input.
\end{abstract}

\section{Introduction}

This paper considers the control of a bilinear system

$$
\dot{x}(t)=A x(t)+u(t) N x(t), \quad x(0)=x_{0},
$$

where $x(t) \in R^{n}$ is the system state vector, $u(t)$ is a scalar control input, and $A \in R^{n \times n}$ and $N \in R^{n \times n}$ are constant square matrices. It is assumed that there exists is a positive definite matrix $Q$ such that

$$
A^{T} Q+Q A=0
$$

In other words, the open-loop system is neutrally stable [1]. Furthermore, the pair $(A, N)$ satisfies the following controllability assumption [2]: there exists an integer $N(\geq n-1)$ such that

$$
\operatorname{span}\left\{a d^{k}(A, N) x_{0}, k=0,1,2, \ldots, N\right\}=R^{n}
$$

for any nonzero $x_{0}$ in $R^{n}$, where $a d^{k}(A, N)$ 's are defined recursively by

$$
\begin{aligned}
a d^{0}(A, N) & =N, \\
a d^{k+1}(A, N) & =A \cdot a d^{k}(A, N)-a d^{k}(A, N) \cdot A,
\end{aligned}
$$

where $k=0,1,2, \ldots \ldots$

Conventionally, quadratic feedback control [3-5] has been proposed for the stabilization of the system (1):

$$
u(t)=-x^{T}(t) Q N x(t)
$$

which ensures global asymptotic stability of the closedloop system. However, it has been shown [6] that the closed-loop system is not exponentially stable, and the state converges as

$$
\|x(t)\| \sim \frac{1}{\sqrt{t}} .
$$

The objective of this work is to introduce a new nonlinear control control which stabilizes the closed-loop system globally and, most importantly, exponentially. The exponential stability results in a much faster time response of the system state than (5); furthermore, it enhances the robustness of the controlled system [7].

\section{Nonlinear Control}

The proposed nonlinear control is as follows:

$$
u(t)=\left\{\begin{array}{ccc}
-\rho \frac{x^{T}(t)}{\|x(t)\|} Q N \frac{x(t)}{\|x(t)\|}, & x(t) \neq 0, \\
0 & , & x(t)=0,
\end{array}\right.
$$

where $\rho$ is a positive control gain, and $Q$ is as in (2). Notice that the control (6) is uniformly bounded for whatever values of the state $x(t)$ :

$$
|u(t)| \leq \rho n q, \quad \forall t>0,
$$

where $q$ and $n$ are respectively the matrix norms of $Q$ and $N$. If the bilinear system (1) is subject to the control constraint 


$$
|u(t)| \leq u_{\max }
$$

the control gain $\rho$ in (6) will have to be chosen within the range:

$$
\rho \in\left(0, \frac{n q}{u_{\max }}\right)
$$

\section{Stability Analysis}

The following lemma can be derived using a contradiction argument based on the controllability assumption (3).

Lemma 1 : If the system (1) satisfies the controllability assumption (3), and there exists a constant vector $x_{0}$ such that for all $t \in[k T, k T+T)$

$$
x_{0}^{T} e^{A^{T}(t-k T)} Q N e^{A(t-k T)} x_{0} \equiv 0,
$$

for any $T>0$, then $x_{0}$ must be the null vector.

Given any time interval length $T>0$, define a scalar function $B(\cdot): S \rightarrow R$ for the controlled system (1) and (6), where $S$ is the unit sphere in $R^{n}$, and $x(t) \neq 0$,

$$
B\left(\frac{x(k T)}{\|x(k T)\|}\right) \triangleq \int_{k T}^{(k+1) T}\left(\frac{x^{T}(t)}{\|x(t)\|} Q N \frac{x(t)}{\|x(t)\|}\right)^{2} d t,
$$

Note that given $Q$ and $N$, the function $B(\cdot)$ is determined by the closed-loop trajectory $x(t) /\|x(t)\|, t \in$ $[k T, k T+T)$, which is in tern uniquely determined by its initial condition $x(k T) /\|x(k T)\|$. Therefore, $B(\cdot)$ in (10) is defined as a function of the initial condition $x(k T) /\|x(k T)\|$. It can be shown that this function has the following property.

Lemma 2 : There exists some positive constant $\beta$ such that

$$
\inf \left[B\left(\frac{x(k T)}{\|x(k T)\|}\right)\right]=\beta>0
$$

where the inf(imum) is taken over all $x(k T) /\|x(k T)\| \in$ $S$ (that is, over all $x(k T) \neq 0$ ).

One can now prove the global exponential stability of the controlled bilinear system.

Theorem : Consider the bilinear system (1) and the nonlinear control (6) subject to the constraint (8). Given any initial condition, the controlled state $x(t)$ converges to zero exponentially.

Proof: Define a Lyapunov function candidate

$$
V(t)=x^{T}(t) Q x(t),
$$

where $Q$ is as in (2). Notice that

$$
x^{T}(t) Q x(t) \leq \bar{\lambda}\|x\|^{2},
$$

where $\bar{\lambda}$ is the maximum eigenvalue of the positive definite matrix $Q$. The time derivative of $V(t)$ along (1) and (6) is given by

$$
\begin{aligned}
\dot{V}(t) & =x^{T}(t)\left(A^{T} Q+Q A\right) x(t)+2 x^{T}(t) Q N x(t) u(t) \\
& =-2 \rho\left(\frac{x^{T}(t)}{\|x(t)\|} Q N \frac{x(t)}{\|x(t)\|}\right)^{2}\|x(t)\|^{2} \leq 0 .(13)
\end{aligned}
$$

Since $V(t)$ is non-increasing, one has

$$
V(k T+T) \leq V(t), \quad \forall t \in[k T, k T+T) .
$$

Integrating the equation (13) from $k T$ to $(k+1) T$ yields

$$
\begin{aligned}
& V(k T+T)-V(k T)= \\
& -2 \rho \int_{k T}^{(k+1) T}\left(\frac{x^{T}(t)}{\|x(t)\|} Q N \frac{x(t)}{\|x(t)\|}\right)^{2} \frac{\|x(t)\|^{2}}{x^{T}(t) Q x(t)} V(t) d t, \\
& \leq-2 \frac{\rho}{\bar{\lambda}} V(k T+T) \int_{k T}^{(k+1) T}\left(\frac{x^{T}(t)}{\|x(t)\|} Q N \frac{x(t)}{\|x(t)\|}\right)^{2} d t, \\
& \leq-2 \frac{\rho \beta}{\bar{\lambda}} V(k T+T),
\end{aligned}
$$

where the first inequality results from (12) and (14), and the second from (11) in Lemma 2. Re-arranging the above equation gives

$$
V(k T+T) \leq \frac{1}{1+2 \rho \beta / \lambda} V(k T)
$$

proving that the Lyapunov function $V(k T)$ decreases exponentially to zero as $k$ approaches infinity, and so does $x(k T)$.

Finally, it remains to show that the continuous state $x(t)$ remains bounded and also converges to zero exponentially. To this end, note from (1) and (7) that

$$
\|\dot{x}(t)\| \leq\left(a+\rho n^{2} q\right)\|x(t)\|,
$$

where $a$ is the matrix norm of the open-loop system matrix $A$. Using (16) and Proposition 1.4.1 in [8], one can derive that the continuous state $x(t)$ is bounded by the discrete state $x(k T)$ by

$$
\|x(t)\| \leq e^{\left(a+\rho n^{2} q\right)(t-k T)}\|x(k T)\|, \quad \forall t \in[k T, k T+T),
$$

and this shows that the continuous state $x(t)$ remains bounded and converges exponentially to zero as the discrete state $x(k T)$ does.

Remark : Notice that the Theorem holds for whatever value of the control saturation limit $u_{\max }>0$ as long as 
the control gain $\rho$ satisfies (8). As a result, even if the control actuator can provide only a small amount of energy (i.e.; a tight saturation limite $u_{\max }$ ), the proposed control can still stabilize the system globally. Such a property is not shared by the conventional quadratic control (4) where the amount of energy required is proportional to the square of $\|x(t)\|$. Hence, large control energy is required if $x(t)$ is initially far from the origin.

\section{Simulation Examples}

Example 1: Consider the bilinear system (1) with

$$
A=\left[\begin{array}{cc}
0 & 2 \\
-2 & 0
\end{array}\right], \quad N=\left[\begin{array}{ll}
0 & 1 \\
1 & 0
\end{array}\right] \text {, }
$$

and the initial condition $x^{T}(0)=[5,-2]$. Figure 1 shows the state response of the system with the conventional quadratic control (4) with $Q=3 I$, and Figure 2 the state response with the new nonlinear control (5) with $\rho=3, Q=I$. It is obvious that the new nonlinear control results in a much faster time response since the state now decays exponentially.

Example 2: Consider same system as in the previous example but with a perturbation on the open-loop system matrix

$$
\Delta A=\left[\begin{array}{cc}
0.4 & 0 \\
0 & 0
\end{array}\right]
$$

The perturbed open-loop system becomes slightly unstable, but still controllable in the sense of (3). The initial condition is $x^{T}(0)=[5,-2]$. Figure 3 shows the closed-loop system becomes unstable under the conventional quadratic control (4). However, the new nonlinear control $(\rho=3, Q=I)$ can still stabilize the perturbed unstable system as is shown in Figure 4

The reason why the new control can stabilize a slightly perturbed system is as follows. Since the closed-loop system with the proposed control is exponential stable, one can show, following Theorem 121 in Chapter 7 of [7], that the exponential stability is retained given any small perturbation in the open-loop system matrix $A$ in (1). Note that such slightly perturbed system may not be stabilized by the conventional quadratic control (4) since it does not provide exponential stability for the nominal closed-loop system.

Finally, note that the constant $\beta$ (defined in (11)) also depends on $\rho$. Consequently, the number $1+2 \rho \beta / \bar{\lambda}$ in (15) may not be a monotonically increasing function of $\rho$. A plot of $1+2 \rho \beta / \bar{\lambda}$ versus $\rho$ is given in Figure 5 for the controlled system in Example 1. The simulation results indicate that the number $1+2 \rho \beta / \bar{\lambda}$ approaches one as $\rho$ approaches zero or infinity. Hence, according to (15), a too small or too large control gain $\rho$ can result in slow state convergence. In other words, there exists an optimal value of the control gain $\rho$, which can best expedites the state convergence. However, at this moment, there is no analytic method to predict this optimal value; it can be searched only throulgh computer simulations.

\section{Conclusions}

In this paper, a new nonlinear control different from the conventional quadratic feedback control is proposed to stabilize a homogeneous-in-the-state bilinear system. The new control results in exponential stability of the closed-loop system, and hence a much faster time response than with the quadratic control.

\section{References}

[1] M. Slemrod, "Stabilization of Bilinear Control Systems with Applications to Nonconservative Problems in Elasticity," SIAM J. Contr. and Optimization, vol.16, pp.131-141, 1978.

[2] M. Vidyasagar, Nonlinear Systems Analysis, Prentice Hall, New Jersey, 1993.

[3] V. Jurdjevic and J. P. Quinn, "Controllability and Stability," Journal of Differential Equations, vol.28, pp.381-389, 1978.

[4] E. P. Ryan and N. J. Buckingham, "On Asymptotically Stabilizing Feedback Control of Bilinear Systems," IEEE Trans. Auto. Contr., vol. AC-28, pp.863-864, 1983

[5] S. N. Singh, "Stabilizing Feedback Controls for Nonlinear Hamiltonian Systems and Nonconservative Bilinear Systems in Elasticity," J. of Dyn. Syst. Meas. and Contr., vol. 104, pp.27-32, 1982.

[6] J. P. Quinn "Stabilization of Bilinear Systems by Quadratic Feedback Controls," Journal of Mathematical Analysis and Applications, vol. 75, pp.66-80, 1980.

[7] F. Callier and C. A. Desoer, Linear System Theory, Springer-Verlag, Hong Kong, 1992.

[8] S. Sastry and M. Bodson, Adaptive Control, Stability, Convergence, and Robustness, Prentice-Hall, London, 1989. 


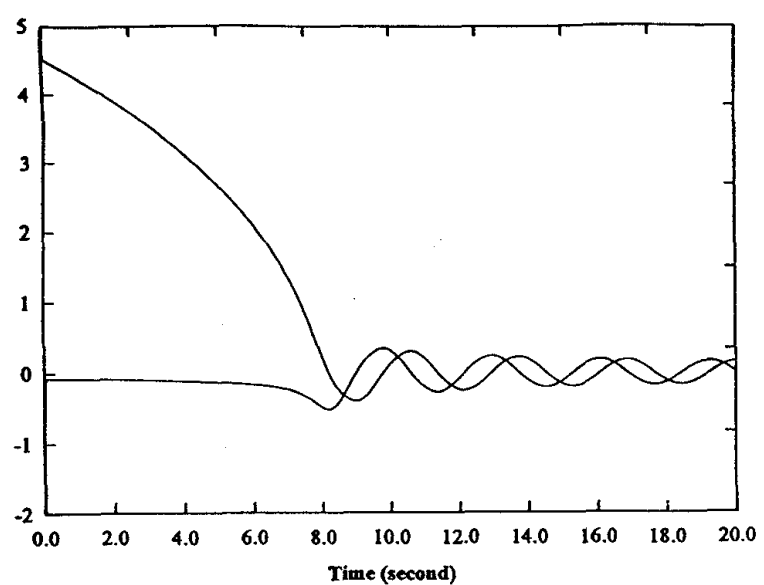

Figure 1. State response with quadratic control

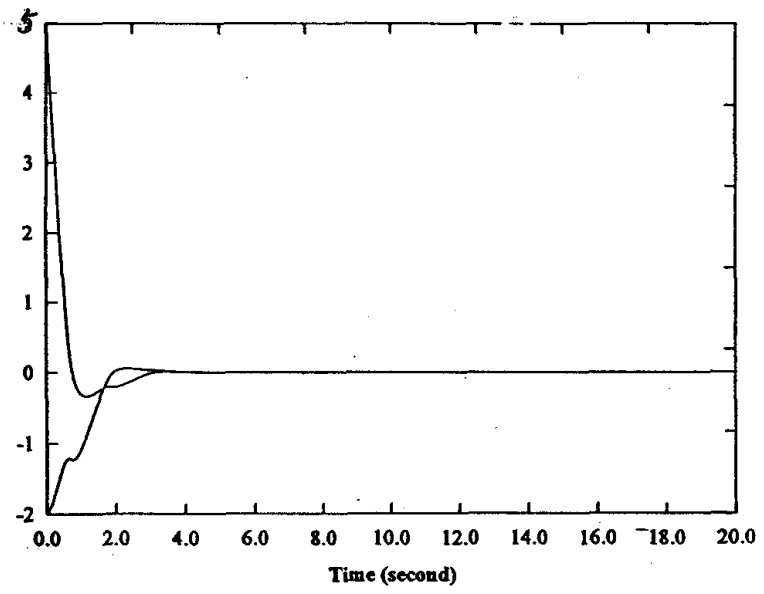

Figure 2. State response with new nonlinear control

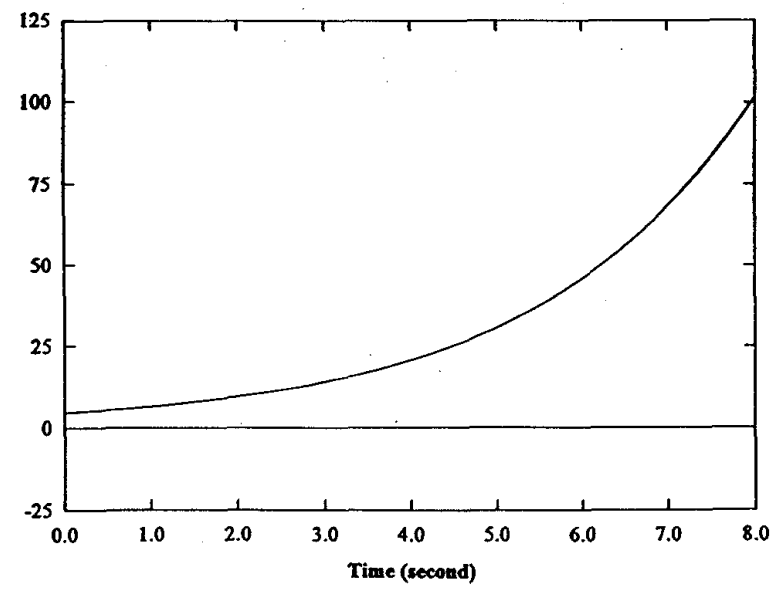

Figure 3. Perturbed response with quadratic control

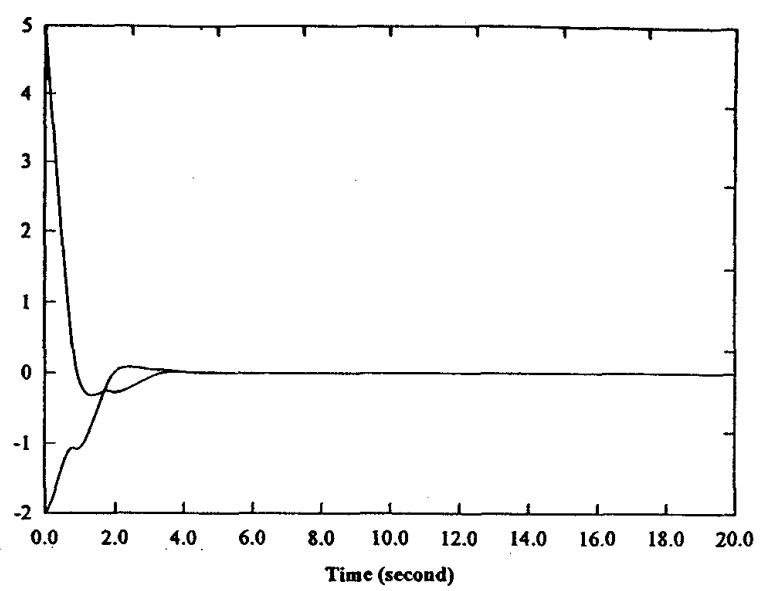

Figure 4. Perturbed response with new nonlinear control

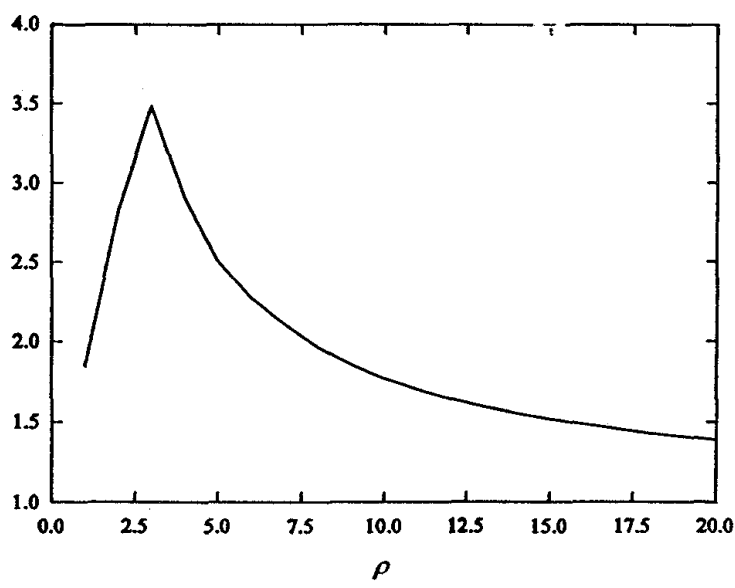

Figure 5. $1+2 \rho \beta / \bar{\lambda}$ versus $\rho$ 